\title{
BILINEAR DISCRETE PAINLEVÉ-II AND ITS PARTICULAR SOLUTIONS
}

\author{
J. SATSUMA \\ Department of Mathematical Sciences \\ University of Tokyo \\ 3-8-1 Komaba, Meguro-ku, Tokyo 153, Japan \\ K. KAJIWARA \\ Department of Electrical Engineering \\ Doshisha University \\ Tanabe, Kyoto 610-03, Japan \\ B. Grammaticos \\ LPN, Université Paris VII \\ Tour 24-14, 5ème étage \\ 75251 Paris, France \\ J. Hietarinta \\ Department of Physics \\ University of Turku \\ 20500 Turku, Finland \\ A. RAMANI \\ CPT, Ecole Polytechnique \\ CNRS, UPR 14 \\ 91128 Palaiseau, France
}

\begin{abstract}
By analogy to the continuous Painlevé II equation, we present particular solutions of the discrete Painlevé II ( $\left.\mathrm{d}-\mathrm{P}_{\mathrm{II}}\right)$ equation. These solutions are of rational and special function (Airy) type. Our analysis is based on the bilinear formalism that allows us to obtain the $\tau$ function for d-P $\mathrm{P}_{\mathrm{II}}$. Two different forms of bilinear $\mathrm{d}-\mathrm{P}_{\mathrm{II}}$ are obtained and we show that they can be related by a simple gauge transformation.
\end{abstract}


The (continuous) Painlevé equations are known as paradigms of integrability [1]. Still, their integrability is of a special type, in the sense that it does not lead to the solution being written in terms of known functions. In fact, the general solution can be obtained only through IST methods (which, in essence, reduce the Painlevé equations to a linear integrodifferential equation) [2]. Although the general solution to the Painlevé equations is essentially transcendental, simple solutions do also exist. In particular for those Painlevé equations that contain free parameters it is possible to find values of these parameters for which a solution can be obtained in terms of special functions [3]. It must be pointed out, though, that this 'elementary' solution does not possess the full dimensionality of integration constants. Typically, the Painlevé equations have (for special values of their parameters) solutions involving one integration constant and which are given in terms of special functions or solutions that do not involve any integration constant and are usually rational expressions.

Let us illustrate this in the case of the second Painlevé equation $\mathrm{P}_{\mathrm{II}}$ :

$$
w^{\prime \prime}=2 w^{3}+x w+\alpha
$$

It is well known that whenever the parameter $\alpha$ is half integer $\mathrm{P}_{\mathrm{II}}$ possesses solutions that can be expressed in terms of Airy functions while for integer $\alpha \mathrm{P}_{\mathrm{II}}$ has solutions of rational type. Thus when $\alpha=1 / 2$ we have $w=-A^{\prime} / A$ with $A$ solution of $A^{\prime \prime}+\frac{x}{2} A=0$. The simplest rational solution is obtained for $\alpha=0$ in which case we find $w=0$. The construction of solutions for higher values of the parameter can be based on the autoBäcklund transform of $\mathrm{P}_{\mathrm{II}}$ [4], that relates the solutions of $\mathrm{P}_{\mathrm{II}}$ corresponding to values $\alpha$ and $\alpha+1$ :

$$
w(\alpha+1)=-w(\alpha)-\frac{1+2 \alpha}{2 w^{2}(\alpha)+2 w^{\prime}(\alpha)+x}
$$

The parity relation $w(-\alpha)=-w(\alpha)$ allows us to reach the negative values of the parameter $\alpha$. Similar results exist for the higher Painlevé equations.

An important result that has been obtained recently concerning these rational solutions. It was shown in [5] that they can be expressed in terms of $\tau$ functions in the form of Wronskian determinants:

$$
\tau_{N}=\left|\begin{array}{cccc}
a_{0} & a_{1} & \cdots & a_{N-1} \\
a_{1} & a_{2} & \cdots & a_{N} \\
\vdots & \vdots & \ddots & \vdots \\
a_{N-1} & a_{N} & \cdots & a_{2 N-2}
\end{array}\right|
$$

where the $a_{k}$ 's satisfy

$$
a_{k}=a_{k-1}^{\prime}+\sum_{j=0}^{k-2} a_{j} a_{k-j-2},
$$

with

$$
a_{0}=x, a_{1}=1
$$


Equations(3) and (4) uniquely determine $a_{k}$. We must point out here that the $a_{k}$ 's satisfy also a linear recursion relation,

$$
a_{k}^{\prime}=2(k-1) a_{k-2}
$$

which however does not suffice for their complete determination. The nonlinear variable $w$ is related to the $\tau$-function though:

$$
w=\left(\log \frac{\tau_{N+1}}{\tau_{N}}\right)^{\prime}
$$

and satisfies $\mathrm{P}_{\mathrm{II}}$ in the form: $w^{\prime \prime}=2 w^{3}-4 x w+4(N+1)$, which is equivalent to (1) up to a scaling of both $w$ and $x$.

In this letter we shall focus on the discrete equivalent of these results. As is well known the Painlevé equations possess discrete analogs [6,7] that are integrable in a sense reminiscent of the continuous case: isomonodromy based on the existence of a Lax pair [8]. Special solutions do also exist. In a series of publications $[9,10,11]$ we have analysed this problem and obtained results for the d-P's from II to V. In what follows we shall return to d-P $\mathrm{P}_{\mathrm{II}}$ :

$$
\bar{u}+\underline{u}=\frac{z u-a}{1-u^{2}}
$$

where we use the notation $u \equiv u_{n}, \bar{u} \equiv u_{n+1}$ and $\underline{u} \equiv u_{n-1}$. Note that $u$ has the parity $u(a)=-u(-a)$ in close analogy to the continuous case. What is known about this equation? In [8] we have obtained the (discrete) Miura and auto-Bäcklund transformations. The former writes:

$$
v=(1-\bar{u})(u+1)-z / 2-\delta / 4
$$

and transforms d-P $\mathrm{P}_{\mathrm{II}}$ to $\mathrm{d}-\mathrm{P}_{34}$ :

$$
(\bar{v}+v)(v+\underline{v})=\frac{4 v^{2}-m^{2}}{v+z / 2+\delta / 4}
$$

where $m=a-\delta / 2$ and $\delta \equiv \bar{z}-z$. The discrete Miura transform (9) is complemented by:

$$
u=\frac{v-\underline{v}-m}{v+\underline{v}}
$$

This means that eliminating $v$ or $u$ between (9) and (11) we can find (8) or (10) respectively. The auto-Bäcklund transformation relating $u(a)$ to $u(a-\delta)$ writes:

$$
u(a-\delta)=-u(a)-\frac{(2 a-\delta)(1+u(a))}{2(u(a)+1)(1-\bar{u}(a))-z-a}
$$

Airy-type solutions were shown to exist. For instance at $a=\delta / 2$ we have $u=-1+\bar{A} / A$ where $A$ is a solution of the discrete Airy equation: $\overline{\bar{A}}-2 \bar{A}+(z / 2+\delta / 4) A=0$. The basic rational solution, as in the continuous case, is $u=0$ for $a=0$. From these 'seed' solutions one can construct higher ones using the Bäcklund transform (12). 
A different approach was presented in [12] where we obtained the general Airy-type solution of $\mathrm{d}_{-} \mathrm{P}_{\mathrm{II}}$ in terms of Casorati determinants. The essential tool in that approach is the bilinear formalism: the solution to the $\mathrm{d}-\mathrm{P}_{\mathrm{II}}$ equation is expressed as a ratio of $\tau$ functions each of which is a Casorati determinant. However, this approach is a 'topdown' one, starting from the determinantal form of the solution and using determinantal (Plücker) identities in order to obtain the equation. This may not be always convenient. In this letter we shall present a different treatment which is also based entirely on the bilinear formalism but starts with the equation and proceeds to the construction of the solution. This approach is particularly convenient for the rational solutions of d-P $\mathrm{P}_{\text {II }}$ as we will show below.

Let us start with the bilinear form of $\mathrm{d}-\mathrm{P}_{\mathrm{II}}$. The bilinear forms of the continuous Painlevé equations have been presented in [13]. It was shown there that starting from the equation (1) and introducing $F, G$ through $w=(\ln F / G)_{x}$ one can obtain a bilinearization in the form:

$$
\begin{gathered}
D^{2} F \cdot G=0 \\
\left(D^{3}-x D-\alpha\right) F \cdot G=0
\end{gathered}
$$

where $D$ is the Hirota operator acting on a dot-product. Its precise definition is the following: $D F \cdot G=\left.\left(\partial_{x}-\partial_{x^{\prime}}\right) F(x) G\left(x^{\prime}\right)\right|_{x=x^{\prime}}=F^{\prime}(x) G(x)-F(x) G^{\prime}(x)$. The quantities $F$ an $G$ are indeed $\tau$ functions for the continuous $\mathrm{P}_{\mathrm{II}}$. In order to construct the discrete analog to (13) one must recall the relation between the parameters of the continuous $\mathrm{P}_{\text {II }}$ (1) and those of the discrete one (8). In fact in order to obtain the continuous limit of d-P II we must take $a=\epsilon^{3} \alpha, z=2+\epsilon^{2} x$ and let $\epsilon \rightarrow 0$ while $u$ becomes $w$. We shall not present here a fully systematic approach of the bilinearization of d-P $\mathrm{II}$. It is clear that the continuous $D$ 's must be replaced by the discrete shift operators $e^{D}$ the action of which is given by $e^{D} f \cdot g=f(n+1) g(n-1)$. To make a long story short, the bilinear form of $\mathrm{d}-\mathrm{P}_{\mathrm{II}}$ reads just:

$$
\begin{gathered}
(\cosh D-1) f \cdot g=0 \\
(\sinh 2 D-z \sinh D-a) f \cdot g=0
\end{gathered}
$$

Equations (14) can be written in a more explicit way as:

$$
\begin{gathered}
\bar{f} \underline{g}+\underline{f} \bar{g}-2 f g=0 \\
\overline{\bar{f}} \underline{\underline{g}}-\overline{\bar{g}} \underline{\underline{f}}-z(\bar{f} \underline{g}-\underline{f} \bar{g})-2 a f g=0
\end{gathered}
$$

The relation of the nonlinear variable $u$ to the $f, g$ is

$$
u=\frac{\bar{f} g}{f g}-1=1-\frac{f \bar{g}}{\overline{f g}}
$$

The resemblance to the continuous case is striking. In fact, one can check that at the continuous limit (14) goes over to (13). Moreover, starting with (9) one can obtain a nonlinear equation for the quantity $u$ that turns out to be exactly the d-P $\mathrm{P}_{\text {II }}(8)$. In order 
to assign a particular meaning to the quantities $f$ and $g$ it is interesting to examine the Miura transform (9). It writes:

$$
v=\frac{\overline{\bar{g}} \underline{\underline{g}}}{\bar{g} g}-z / 2-\delta / 4
$$

Next we use the symmetry property $u(a)=-u(-a)$ rewritten in terms of the parameter $m$ : we have $u(m+\delta / 2)=-u(-(m+\delta)+\delta / 2)$. Thus the Miura transform (9) can be used to construct not only the solution $v$ corresponding to $m$ but also the solution $w$ corresponding to $-(m+\delta)$ and thanks to the invariance of (10) with respect to $m \rightarrow-m$ this solution coincides with the one at $(m+\delta)$. Implementing the Miura transform we find:

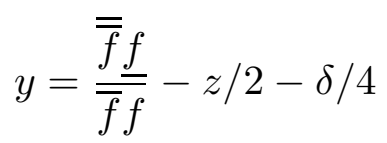

At this stage the situation becomes clear: $g$ is related to $m=a-\delta / 2$ while $f$ goes with $m^{\prime}=a+\delta / 2$. In fact the $g$ and $f$ are $\tau$ functions corresponding to two consecutive values of the parameter $a$ with difference $\delta$.

We now turn to the construction of the rational solutions of (14). As we have already explained in [9] equation (8) possesses rational solutions that do not contain any free parameter for values of the parameter $a$ that are integer multiples of $\delta$. Thus when $a=0$ we find $u=0$ as a solution, while for $a=\delta$ we obtain $u=\delta /(z-2)$. In order to construct the rational solutions for higher values of $a$ one can use the auto-Bäcklund transform(12). However the use of $\tau$ functions leads to this result in a more natural way. Let us first rewrite $\mathrm{d}-\mathrm{P}_{\mathrm{II}}$ so as to introduce directly the parameters related to the continuous limit $\left(\delta=-\epsilon^{3}\right)$ :

$$
\bar{u}+\underline{u}=\frac{u\left(2-\epsilon^{3} n\right)+\epsilon^{3}(N+1)}{1-u^{2}}
$$

In terms of the $\tau$-functions, $u$ can be written simply as:

$$
u=\frac{\bar{\tau}_{N+1} \underline{\tau}_{N}}{\tau_{N+1} \tau_{N}}-1
$$

We start with $\tau_{0}=1$ (and also $\tau_{-1}=1$ ) and $\tau_{1}=n$. We then use the auto-Bäcklund transformation in order to compute the $\tau$ 's for higher $N$ 's. We obtain thus $\left(\nu \equiv \epsilon^{-3 / 2}\right)$ :

$$
\begin{gathered}
\tau_{2}=n\left(n^{2}-1\right)-4 \nu^{2} \\
\tau_{3}=n^{2}\left(n^{2}-1\right)\left(n^{2}-4\right)-4 n\left(5 n^{2}-8\right) \nu^{2}-80 \nu^{4}
\end{gathered}
$$

and so on. What is really interesting is that we can, just as in the continuous case, cast these $\tau$-functions in the form of a determinant. We find thus for $\tau_{3}$ the expression:

$$
\tau_{3}=\left|\begin{array}{ccc}
n & 2 \nu & n(n+2) \\
2 \nu & n^{2}-1 & 8 \nu\left(n+\frac{1}{2}-\frac{3}{4} \sigma\right) \\
n(n-2) & 8 \nu\left(n-\frac{1}{2}+\frac{3}{4} \sigma\right) & 2 n\left(n^{2}-4\right)+20 \nu^{2}
\end{array}\right|
$$


where $\sigma$ is just a sign, $\sigma= \pm 1$. Higher $\tau$ 's can be (and have been) computed and cast in the form of a Casorati determinant.

$$
\tau=\left|\begin{array}{ccccc}
a_{0}(n) & a_{1} & a_{2}(n+1) & a_{3}^{-}(n+2) & \ldots \\
a_{1} & a_{2}(n) & a_{3}^{+}(n) & a_{4}(n+1) & \ldots \\
a_{2}(n-1) & a_{3}^{-}(n) & a_{4}(n) & a_{5}^{+}(n) & \ldots \\
a_{3}^{+}(n-2) & a_{4}(n-1) & a_{5}^{-}(n) & a_{6}(n) & \ldots \\
\vdots & \vdots & \vdots & \vdots & \ddots
\end{array}\right|
$$

where the following relation holds $a_{k}^{ \pm}(n, \sigma)=a_{k}\left(n \pm \frac{1}{2}, \pm \sigma\right)$ and:

$$
\begin{gathered}
a_{0}=n \\
a_{1}=2 \nu \\
a_{2}=n^{2}-1 \\
a_{3}=8 \nu\left(n-\frac{3}{4} \sigma\right) \\
a_{4}=2 n\left(n^{2}-4\right)+20 \nu^{2} \\
a_{5}=32 \nu\left(\left(n+\frac{3}{4} \sigma\right)^{2}-\frac{19}{16}\right) \\
a_{6}=5\left(n^{2}-1\right)\left(n^{2}-9\right)+200 \nu^{2} n \\
=128 \nu\left(\left(n-\frac{3}{4} \sigma\right)^{3}-\frac{73}{16}\left(n-\frac{3}{4} \sigma\right)+\frac{15}{4} \nu^{2}-\frac{3}{64} \sigma\right) \\
a_{8}=14 n\left(n^{2}-4\right)\left(n^{2}-16\right)+4 \nu^{2}\left(350 n^{2}-557\right)
\end{gathered}
$$

What is lacking at the present stage is the equivalent of relations $(3,4,5)$ that allows the construction of the matrix elements. It is not very difficult to obtain the equivalent of the (linear) differential relation (5) although some complications appear because of the parity dependence of the matrix elements. We have, for even $k$ :

$$
a_{k}(n+1)-a_{k}(n-1)=4(k-1) a_{k-2}(n)
$$

while for odd $k$ we find:

$$
a_{k}(n+1, \pm \sigma)-a_{k}(n-1, \pm \sigma)=4(k-1) a_{k-2}(n, \mp \sigma)
$$

However the equivalent of the nonlinear relation (3) has not been found in the discrete case. Still, its existence would lead only to a practical simplification. The important point is that the $\tau$ functions (which are the fundamental objects) can be computed in an algorithmic way. In fact, instead of using the Bäcklund for $u$, equation (12), we can derive a Bäcklund relation directly for the $\tau$-functions:

$$
\tau_{N+1} \bar{\tau}_{N-1}=2 \nu^{2} \overline{\bar{\tau}}_{N \underline{\tau}_{N}}+\bar{\tau}_{N} \tau_{N}\left(n-N-2 \nu^{2}\right)
$$




$$
\bar{\tau}_{N+1} \tau_{N-1}=2 \nu^{2} \overline{\bar{\tau}}_{N} \underline{\tau}_{N}+\bar{\tau}_{N} \tau_{N}\left(N+n+1-2 \nu^{2}\right)
$$

Next we turn to an important question concerning the comparison of the present results with those of our work on the Airy-type solutions of d-P $\mathrm{P}_{\mathrm{II}}$. In [12], we have obtained the following bilinear expressions:

$$
\begin{gathered}
\tau_{N+1}^{n-1} \tau_{N-1}^{n+2}=\tau_{N}^{n-1} \tau_{N}^{n+2}-\tau_{N}^{n} \tau_{N}^{n+1} \\
\tau_{N+1}^{n+2} \tau_{N}^{n+1}-2 \tau_{N+1}^{n+1} \tau_{N}^{n+2}+(p n+q) \tau_{N+1}^{n} \tau_{N}^{n+3}=0 \\
\tau_{N+1}^{n+1} \tau_{N-1}^{n+2}=-(p(n+2 N)+q) \tau_{N}^{n+2} \tau_{N}^{n+1}+(p n+q) \tau_{N}^{n} \tau_{N}^{n+3}
\end{gathered}
$$

and with the dependent variable transformation

$$
w_{n}=\frac{\tau_{N+1}^{n+1} \tau_{N}^{n}}{\tau_{N+1}^{n} \tau_{N}^{n+1}}-1
$$

we obtain the standard nonlinear form of $\mathrm{d}-\mathrm{P}_{\mathrm{II}}$ :

$$
w_{n+1}+w_{n-1}=\frac{(2 p n+(2 N-1)+2 q) w_{n}-(2 N+1) p}{1-w_{n}^{2}}
$$

We remark that equation (K19) corresponds to $a=(2 N+1) p$ while the $a$ of equation (19) is $-\epsilon^{3}(N+1)$. The interpretation of this value of $a$ is the following. Equation (K19), and also (K15-18), are written specifically for the case of Airy-type solutions, i.e. half-integer values of $a / \delta$ and the $\tau_{N}$ of $(\mathrm{K} 18)$ is associated to $a=(2 N+1) p$. In the present paper, the formalism of (15ab) is quite general and does not depend on the type of solution considered. (Only when rational solutions are considered does $N$ have to be taken as an integer). If we wish to compare the bilinear expressions (K15-17) and $(15 \mathrm{ab}, 26,27)$ we must take into account the shift in $N$. The simplest way to do this is to rewrite (K19) as:

$$
w_{n+1}+w_{n-1}=\frac{(2 p(n+N)+2 q) w_{n}-2(N+1) p}{1-w_{n}^{2}}
$$

Comparing (K19') to (19) we obtain $p=-\epsilon^{3} / 2=-1 / 2 \nu^{2}$ and $q=1+N / 2 \nu^{2}$. Next, let us remark that there exists a further small difference in the definitions of the $\tau$ 's in the two papers: what is called $\tau_{M}(m)$ in [12] corresponds in this paper to a $\tau$-function, which we will here denote by $\kappa_{M}(m+M-N-1)$, i.e. with the same lower index but an upper index $(m+M-N-1)$, so that (K18) becomes identical to our equation (20). We can now rewrite (K15-17) in terms of the $\kappa$ 's:

$$
\begin{gathered}
\kappa_{N+1}^{n-1} \kappa_{N-1}^{n}=\kappa_{N}^{n-2} \kappa_{N}^{n+1}-\kappa_{N}^{n-1} \kappa_{N}^{n} \\
\kappa_{N+1}^{n+2} \kappa_{N}^{n}-2 \kappa_{N+1}^{n+1} \kappa_{N}^{n+1}+\left(1+\frac{N-n}{2 \nu^{2}}\right) \kappa_{N+1}^{n} \kappa_{N}^{n+2}=0 \\
\kappa_{N+1}^{n+1} \kappa_{N-1}^{n}=-\left(1-\frac{N+n+1}{2 \nu^{2}}\right) \kappa_{N}^{n+1} \kappa_{N}^{n}+\left(1+\frac{N-n}{2 \nu^{2}}\right) \kappa_{N}^{n-1} \kappa_{N}^{n+2}
\end{gathered}
$$


Clearly, equation (29) involving only two consecutive lower indices is analogous to our equation (15a). The difference in form between these two equations can be explained by a gauge transformation between our $\tau$ 's and the $\kappa$ 's. Defining a gauge function $h$ we put

$$
\kappa_{M}^{m}=h_{M}^{m} \tau_{M}^{m}
$$

In order for $w_{n}$ to coincide with $u_{n}$ we must have $\frac{h_{N+1}^{n+1} h_{N}^{n-1}}{h_{N+1}^{n} h_{N}^{n}}=1$. This can be integrated once to $h_{N}^{n+1}=h_{N}^{n} j(n-N)$ and then further integrated to $h_{N}^{n}=J(n-N) \phi(N)$ such that $j(m)=J(m+1) / J(m)$. Introducing the gauge $h$, equation (29) down-shifted once in $n$ becomes:

$$
h_{N+1}^{n+1} h_{N}^{n-1} \tau_{N+1}^{n+1} \tau_{N}^{n-1}-2 h_{N+1}^{n} h_{N}^{n} \tau_{N+1}^{n} \tau_{N}^{n}+\left(1+\frac{N+1-n}{2 \nu^{2}}\right) h_{N+1}^{n-1} h_{N}^{n+1} \tau_{N+1}^{n-1} \tau_{N}^{n+1}=0
$$

All the $\phi$ 's factor out, and (32) coincides with (15a) provided a single relation is satisfied, which defines the gauge function $j$ :

$$
j(n-N-2)=j(n-N)\left(1+\frac{N+1-n}{2 \nu^{2}}\right)
$$

Next we turn to (28), upshift it once in $n$, introduce $h$ and compare it to (26). We remark that thanks to (33) the ratio of the $j$ 's create exactly the right $n-N$-dependent terms in (26). We are left with one condition that defines $\phi$ :

$$
\frac{\phi(N+1) \phi(N-1)}{\phi(N)^{2}}=\frac{1}{2 \nu^{2}}
$$

Finally one can check that (30) is now identical to (27). Thus the two bilinear formulations of $\mathrm{d}-\mathrm{P}_{\mathrm{II}}$ are equivalent, provided one introduces the right gauge.

In this paper we have introduced a bilinear formalism for the description of the discrete Painlevé-II equation. This approach has turned out to be very convenient for the expression of the rational solutions that $\mathrm{d}-\mathrm{P}_{\mathrm{II}}$ possesses for particular values of its parameters. From the analysis presented above it is clear that the choice of the appropriate gauge for the description of the equations depends crucially on the type of the solutions one wishes to examine. The novel feature concerning the rational solutions of $\mathrm{d}_{-} \mathrm{P}_{\mathrm{II}}$ is that they can be given in the form of Casorati determinants, a property that is not yet fully explored even in the continuous case. We expect that the bilinear formalism will be extremely useful in the study of the remaining discrete Painlevé equations and will help establish a perfect parallel between the discrete and the continuous case.

REFERENCES.

[1] P. Painlevé, Acta Math. 25 (1902) 1; B. Gambier, Acta Math. 33 (1910) 1.

[2] M.J. Ablowitz, and H. Segur, Phys. Rev. Lett. 38 (1977) 1103; H. Flashka and A.C. Newell, Com. Math. Phys. 76 (1980) 67; A.S. Fokas and X. Zhou, Comm. Math. Phys. 142 (1991) 313. 
[3] V.A. Gromak and N.A. Lukashevich, The analytic solutions of the Painlevé equations, (Universitetskoye Publishers, Minsk 1990), in Russian.

[4] A.S. Fokas and M.J. Ablowitz, J. Math. Phys. 23 (1982) 2033.

[5] K. Kajiwara and Y. Ohta, in preparation.

[6] A. Ramani, B. Grammaticos and J. Hietarinta, Phys. Rev. Lett. 67 (1991) 1829.

[7] M. Jimbo, T. Miwa and K. Ueno, Physica D 2 (1981) 306.

[8] B. Grammaticos and A. Ramani, Discrete Painlevé equations: derivation and properties, in Applications of analytic and geometric methods to nonlinear differential equations, P.A. Clarkson ed., NATO ASI C413 (1993) 299.

[9] A. Ramani and B. Grammaticos, J. Phys. A: Math. Gen. 25 (1992) L633.

[10] B. Grammaticos, F.W. Nijhoff, V. Papageorgiou, A. Ramani and J. Satsuma, Phys. Lett. A185 (1994) 446.

[11] K.M. Tamizhmani, B. Grammaticos and A. Ramani, Lett. Math. Phys. 29 (1993) 49.

[12] K. Kajiwara, Y. Ohta, J. Satsuma, B. Grammaticos and A. Ramani, J. Phys. A 27 (1994) 915.

[13] J. Hietarinta and M.D. Kruskal, Hirota forms for the six Painlevé equations from singularity analysis, in Painlevé transcendents: their asymptotics and physical applications, D. Levi and P. Winternitz eds, NATO ASI series B278, Plenum 1992, p.175. 